\title{
Liquid Wax Ester Production by Euglena gracilis
}

\author{
Yoshiki TANI, Masakazu OKUMURA* \\ and Shigeru II* \\ Research Center for Cell and Tissue Culture, Faculty of \\ Agriculture, Kyoto University, Kyoto 606, Japan \\ * Research Center, Harima Chemicals, Inc., \\ Kakogawa, Hyogo 675, Japan \\ Received August 8, 1986
}

\begin{abstract}
Euglena gracilis strain $\mathrm{Z}$ produced liquid wax esters when it was incubated anaerobically in the presence of unsaturated fatty acids after aerobic cultivation for 3 days on a glucose-peptone medium. On anaerobic incubation for 6 days with the supplementation of $1 \%$ of oleic acid, linoleic acid, $\alpha$-linolenic acid or ricinoleic acid, $6.9 \sim 8.4 \mathrm{~g} /$ liter of wax esters was accumulated in the cells. The yields increased to about $50 \%$ of the dry cell weight, and all of the wax esters were in the liquid state at room temperature. Gas-liquid chromatographic analysis showed that the unsaturated fatty acids provided not only the acyl moiety of the wax esters but also the alkoxy one, which was formed through reduction to the corresponding fatty alcohols and then incorporated into the wax esters.
\end{abstract}

Some natural wax esters obtained from sperm whale oil and jojoba oil, consisting of $\mathrm{C}_{28}$ through $\mathrm{C}_{40}$ saturated, mono- and diunsaturated wax esters, and $\mathrm{C}_{36}$ through $\mathrm{C}_{44}$ diunsaturated wax esters, respectively, are commercially used as specialized lubricants and as ingredients of cosmetics, for example. However, there are currently problems as to the supplies of these oils, especially sperm whale oil, of which the production is limited due to legislation as to the extent of whaling.

The microbial production of wax esters was reported, using $n$-paraffin-assimilating bacteria such as Micrococcus cerificans, ${ }^{1)}$ Nocardia sp., ${ }^{2)}$ Mycobacterium sp. ${ }^{3)}$ and Acinetobactor sp. ${ }^{4,5)}$ But most of them produce saturated, rather than unsaturated, wax esters. DeWitt et al. exceptionally reported ${ }^{5)}$ that Acinetobactor sp. HO1-N can synthesize liquid waxes, bearing close chemical similarities to those of sperm whale and jojoba oils, from $n$-alkanes $\left(\mathrm{C}_{16}\right.$ through $\left.\mathrm{C}_{20}\right)$.

Inui et al. ${ }^{6 \sim 8)}$ reported that shifting of the growth of Euglena gracilis from under aerobic to anaerobic conditions could result in significant conversion of a storage polysac- charide, paramylon ( $\beta-1,3$ glucan), to wax esters. The latter were saturated wax esters, primarily with carbon chain lengths of 28,27 , 29 and 30.

In this paper, we report the ability of $E$. gracilis to produce liquid waxes efficiently, through the conversion of unsaturated fatty acids, such as oleic acid, added to the medium into wax esters.

\section{MATERIALS AND METHODS}

Microorganism. E. gracilis strain $\mathrm{Z}$ was used.

Chemicals. Oleic acid $\left(94.7 \%\right.$ oleic acid $\left(\mathrm{C}_{18: 1}\right), 1.7 \%$ stearic and $\left(\mathrm{C}_{18: 0}\right)$ and $1.5 \%$ linoleic acid $\left.\left(\mathrm{C}_{18: 2}\right)\right)$ was purchased from Nippon Oil \& Fat Co., Ltd. Linoleic acid $(88.7 \%$ linoleic acid, $9.8 \%$ oleic acid, $0.6 \%$ palmitic acid $\left(\mathrm{C}_{16: 0}\right)$ and $0.5 \%$ linolenic acid $\left.\left(\mathrm{C}_{18: 3}\right)\right)$ and ricinoleic acid $\left(81.8 \%\right.$ ricinoleic acid $\left(\mathrm{C}_{18: 1 \mathrm{OH}}\right), 6.6 \%$ linoleic acid, $5.7 \%$ oleic acid, $1.7 \%$ myristic acid $\left(\mathrm{C}_{14: 0}\right)$ and $1.2 \%$ palmitic acid) of reagent grade from Wako Pure Chemical Industries, Ltd. were used. Reagent grade $\alpha$-linolenic acid (72.7\% $\alpha$-linolenic acid, $19.7 \%$ linoleic acid and $7.1 \%$ oleic acid) was from Nakarai Chemicals, Ltd.

Medium and cultivation. E. gracilis was grown on a glucose-peptone medium containing appropriate amounts 
of glucose and Polypepton (Daigo), $0.25 \mathrm{~g}$ of $\left(\mathrm{NH}_{4}\right)_{2} \mathrm{SO}_{4}$, $0.25 \mathrm{~g}$ of $\mathrm{KH}_{2} \mathrm{PO}_{4}, 0.6 \mathrm{~g}$ of $\mathrm{MgCO}_{3}, 0.12 \mathrm{~g}$ of $\mathrm{CaCO}_{3}$, $50 \mathrm{mg}$ of $\mathrm{Na}_{2} \cdot$ EDTA, $50 \mathrm{mg}$ of $\mathrm{FeSO}_{4}\left(\mathrm{NH}_{4}\right)_{2} \mathrm{SO}_{4} \cdot 6 \mathrm{H}_{2} \mathrm{O}$, $18 \mathrm{mg}$ of $\mathrm{MnSO}_{4} \cdot \mathrm{H}_{2} \mathrm{O}, 25 \mathrm{mg}$ of $\mathrm{ZnSO}_{4} \cdot 7 \mathrm{H}_{2} \mathrm{O}, 2.5 \mathrm{mg}$ of thiamine $\cdot \mathrm{HCl}$ and $0.5 \mathrm{~g}$ of cyanocobalamin in $1000 \mathrm{ml}$ of tap water, $\mathrm{pH}$ 4.5. A preculture $(5 \mathrm{ml})$ in the glucosepeptone medium was transferred to $100 \mathrm{ml}$ of the same medium in a 500 - $\mathrm{ml}$ shaking flask. Cultivation was aerobically carried out in the dark at $28^{\circ} \mathrm{C}$. After cultivation for 3 days until the late logarithmic phase, the whole of the culture broth after adjustment to $\mathrm{pH} 6.5$ was transferred to a round-bottomed flask, and then an adequate amount of an unsaturated fatty acid was added. The cultures were immediately brought to anaerobic conditions by bubbling $\mathrm{N}_{2}$ gas and then stirred at $27^{\circ} \mathrm{C}$. After the prescribed incubation, the cells were harvested by centrifugation $(4000 \times g)$ and then washed with distilled water. Wax ester production was followed by means of gas-liquid chromatographic analysis. Growth was followed turbidimetrically at $610 \mathrm{~nm}$.

Analyses. The harvested cells were suspended in a 10times volume of chloroform-methanol $(1: 1, \mathrm{v} / \mathrm{v})$, and then sonicated at $19.5 \mathrm{kHz}$ for $10 \mathrm{~min}$. The homogenate was centrifuged at $8000 \times g$ for $20 \mathrm{~min}$, and the solvent layer was separated from the aqueous layer and then evaporated in vacuo. Wax esters were isolated from the residual fatty acid and other lipid components in the extract by loading the extract onto a glass column packed with Wakogel C-200. $n$-Hexane-ethyl acetate $(95: 5, \mathrm{v} / \mathrm{v})$ was used as the eluent. The effluent from the column was checked by thin layer chromatography on a Silica Gel 60 aluminum sheet (Merck) with a solvent system of $n$ hexane-ethyl acetate $(90: 10, \mathrm{v} / \mathrm{v})$. As the thin layer chromatographic system clearly resolved the lipid components, wax esters $(R f=0.71)$, triglycerides $(R f=0.24)$ and free fatty acids $(R f=0.04)$, the fractions which showed a single spot with an $R f$ of around 0.71 were collected and pooled as the wax ester fraction.

For determination of the fatty acid and fatty alcohol compositions of the wax esters, the separated wax esters were saponified in ethanolic $1 \mathrm{~N} \mathrm{KOH}$ by refluxing for $2 \mathrm{hr}$ under a stream of $\mathrm{N}_{2}$ gas. Fatty alcohol moieties were extracted with petroleum ether from the hydrolyzate at alkaline pH. Fatty acid moieties in the residual ethanolwater layer were extracted with ethyl ether after adjustment of the $\mathrm{pH}$ to 1.0 with conc. $\mathrm{HCl}$. The extracted fatty acids were methylated with a diazomethane ether solution prepared according to the method of De Boer and Backer. ${ }^{9)}$ The fatty acid methyl esters and fatty alcohols were determined by gas-liquid chromatography.

Gas-liquid chromatography. For identification and determination of the constituent wax esters, wax esters, fatty acid methyl esters and fatty alcohols were analyzed with a Shimadzu GC-7AG gas chromatograph equipped with a flame ionization detector. For the wax esters, it was operated at $280^{\circ} \mathrm{C}$ with a glass column $(0.3 \times 150 \mathrm{~cm})$ packed with $5 \%$ Silicone GE SE-30 on $60 \sim 80$ mesh Shimalite $\mathrm{W}$. On this analysis, a linear relationship between the carbon numbers of wax esters and their retention times plotted on a logarithmic scale was obtained. For the fatty acid methyl esters and fatty alcohols, a glass column $(0.3 \times 200 \mathrm{~cm})$ packed with $20 \%$ diethyleneglycol succinate on $60 \sim 80$ mesh Chromosorb W was used at $200^{\circ} \mathrm{C}$.

\section{RESULTS AND DISCUSSION}

\section{Effect of oleic acid on the formation of wax esters}

After aerobic cultivation on a glucosepeptone medium, in which the concentrations of glucose and Polypepton were $2 \%$ and $0.5 \%$, respectively, for 3 days until near the stationary phase, anaerobic incubation was carried out with and without the supplementation of $1 \%$ oleic acid for 4 days (Fig. 1). Wax ester formation was initiated by shifting the cultivation conditions from aerobic to anaerobic, as reported by Inui et al. ${ }^{6 \sim 8)}$ It was pointed out that cells supplemented with oleic acid accumulated a larger amount of wax esters than non-supplemented cells. Figure 2 shows the gas-liquid chromatograms of the wax esters produced on 4 days' anaerobic incubation with

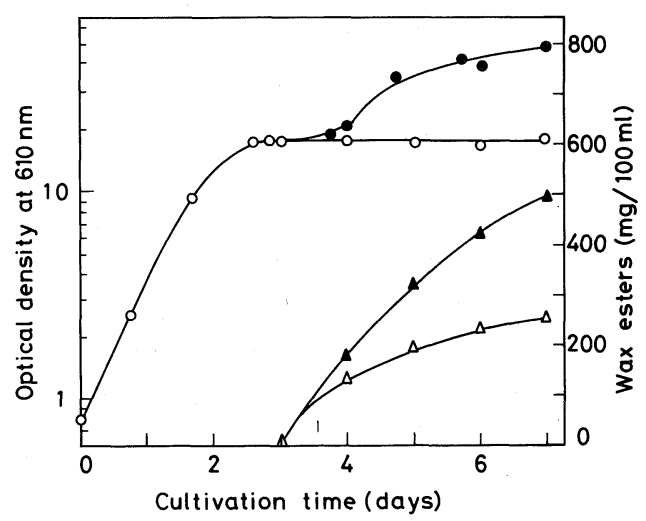

FIG. 1. Growth and Wax Ester Accumulation of $E$. gracilis Strain Z.

The aerobic cultural conditions were changed to anaerobic ones after 3 days' cultivation, when oleic acid was added. $\bigcirc$, growth without supplementation of oleic acid; $\mathbf{O}$ growth with supplementation of $1 \%$ oleic acid; $\triangle$, accumulation of wax esters in cells without supplementation; $\mathbf{\Delta}$, accumulation of wax esters in cells supplemented with $1 \%$ oleic acid. 


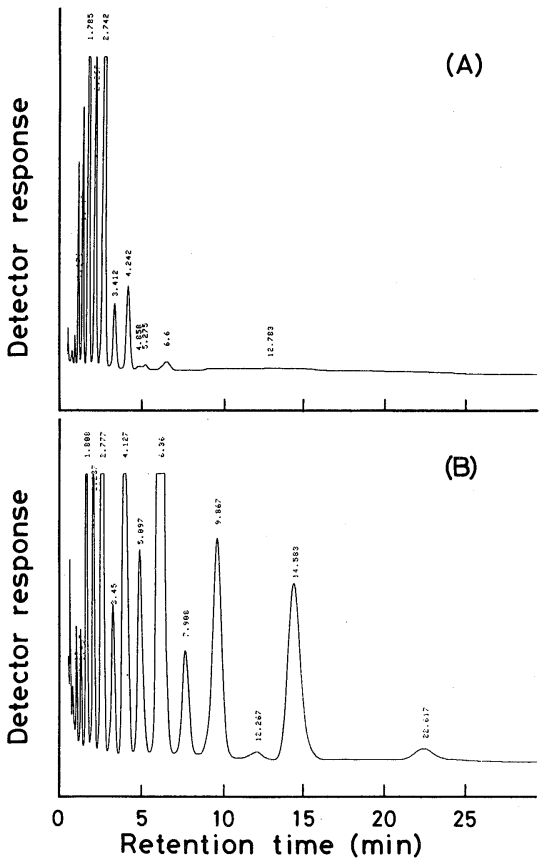

Fig. 2. Gas-liquid Chromatograms of the Wax Esters Produced on 4 Days' Anaerobic Incubation with (B) and without (A) the Supplementation of $1 \%$ Oleic Acid.

The numerical values in the figure are the retention times in $\min$.
Table I. Compositions of the Wax Esters SyNTHESIZED ON ANAEROBIC INCUBATION

After 3 days' aerobic cultivation on a glucose-peptone medium, in which the concentrations of glucose and peptone were $2 \%$ and $0.5 \%$, respectively, anaerobic incubation was carried out for 4 days with and without supplementation of $1 \%$ oleic acid.

\begin{tabular}{|c|c|c|}
\hline \multirow{2}{*}{$\begin{array}{c}\text { Carbon } \\
\text { number of } \\
\text { wax esters }\end{array}$} & \multicolumn{2}{|c|}{$\begin{array}{l}\text { Composition of wax esters } \\
\text { in relative amounts }(\%)\end{array}$} \\
\hline & $\begin{array}{c}\text { No } \\
\text { supplementation }\end{array}$ & $\begin{array}{l}\text { Supplemented } \\
\text { with oleic acid }\end{array}$ \\
\hline 23 & - & - \\
\hline 24 & 5.0 & 0.6 \\
\hline 25 & 7.7 & 0.9 \\
\hline 26 & 25.5 & 4.4 \\
\hline 27 & 14.8 & 3.8 \\
\hline 28 & 33.6 & 12.0 \\
\hline 29 & 4.5 & 2.7 \\
\hline 30 & 6.9 & 9.5 \\
\hline 31 & 0.5 & 5.4 \\
\hline 32 & 1.5 & 31.4 \\
\hline 33 & - & 3.8 \\
\hline 34 & - & 10.9 \\
\hline 35 & - & 0.5 \\
\hline 36 & - & 12.8 \\
\hline 37 & - & - \\
\hline 38 & - & 1.3 \\
\hline
\end{tabular}

Table II. Distributions of Acyl and Alkoxy Groups in the Wax Esters Synthesized on Anaerobic InCubation With and without Supplementation of Oleic Acid

\begin{tabular}{|c|c|c|c|c|}
\hline \multirow{3}{*}{ Component* } & \multicolumn{4}{|c|}{ Relative amounts of acyl and alkoxy groups $(\%)$} \\
\hline & \multicolumn{2}{|c|}{ No supplementation } & \multicolumn{2}{|c|}{ Supplemented with oleic acid } \\
\hline & Acyl & Alkoxy & Acyl & Alkoxy \\
\hline $11: 0$ & 0.3 & 0.6 & - & - \\
\hline $12: 0$ & 16.2 & 19.3 & 5.7 & 14.2 \\
\hline $13: 0$ & 17.4 & 14.8 & 2.0 & 4.7 \\
\hline $14: 0$ & 55.4 & 56.1 & 22.0 & 52.1 \\
\hline $15: 0$ & 2.0 & 2.0 & 0.3 & 2.5 \\
\hline $16: 0$ & 7.0 & 7.2 & 2.1 & 8.8 \\
\hline $17: 0$ & 1.4 & - & 0.4 & - \\
\hline $18: 0$ & 0.3 & - & 0.8 & - \\
\hline $16: 1$ & - & - & 0.5 & - \\
\hline $18: 1$ & - & - & 63.3 & 17.5 \\
\hline $18: 2$ & - & - & 1.0 & - \\
\hline $20: 1$ & - & - & 0.3 & 0.3 \\
\hline
\end{tabular}

* The numerical values on the left side are the carbon numbers, and those on the right side the numbers of unsaturations. 
and without supplementation of $1 \%$ oleic acid. It was found that a large amount of components with retention times of more than $6 \mathrm{~min}$, which were hardly detected in nonsupplemented cells, was synthesized when oleic acid was added to the medium. The composition, and the acyl and alkoxy moiety distribution of each wax ester are shown in Tables I and II, respectively. The carbon chain length of the wax esters in non-suspplemented cells ranged from $24 \sim 32$, and unsaturated constituents were not detected at all. On the other hand, when $1 \%$ oleic acid was added, oleic acid was the principal constituent of the acyl moiety, and oleyl alcohol occupied about $17 \%$ of the alkoxy moiety in the wax esters.

Wax ester fermentation by Euglena was reported by Inui et al. ${ }^{6,8)}$ It was proposed that the fermentation was initiated with decomposition of a storage polysaccharide, paramylon ( $\beta-1,3$ glucan), to acetyl-CoA through glucose and pyruvate on shifting of the culture conditions from aerobic to anaerobic. Subsequent de novo synthesis of fatty acids with average carbon chains of 13.9 from acetyl-CoA in mitochondria and partial reduction of the fatty acids to the corresponding fatty alcohols provided wax esters in microsomes. It should be emphasized that no desaturation of carbon chains occurs during this wax ester synthesis.

In the case of anaerobic incubation with supplementation of oleic acid, it is supposed that oleic acid was taken up into the cells and utilized through the same pathway of wax ester fermentation as described above at the same time. The microsomal fatty acyl-CoA reductase of Euglena, which is highly specific for myristoyl-CoA and palmitoyl-CoA, was reported by Kolattukudy. ${ }^{10)}$ The results of the present study show that the enzyme might reduce oleoyl-CoA. Consequently, the unsaturated acyl and alkoxy groups were incorporated with the saturated ones from paramylon into the wax esters.

\section{Cultural conditions for E. gracilis}

E. gracilis can grow photoautotrophically in the mineral salt medium, and also heterotrophically in several organic growth media, such as Koren-Hutner medium, ${ }^{11)}$ containing glucose, malate and glutamate as the main carbon sources. To improve the productivity of liquid wax esters, the cultural conditions for E. gracilis strain $\mathrm{Z}$ were investigated. A glucose-peptone medium was selected as the culture medium.

The growth curves for E. gracilis strain Z on the glucose-peptone medium with different concentrations of glucose are shown in Fig. 3. Good growth was obtained at a glucose con-

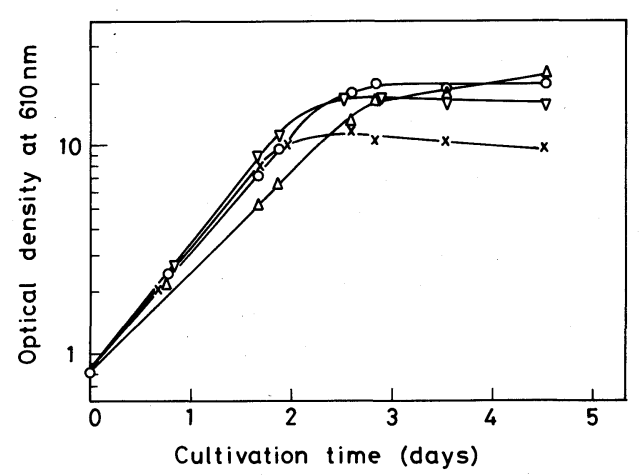

Fig. 3. Growth of E. Gracilis Strain Z on Glucosepeptone Medium.

The concentrations of glucose were: $\times, 1 \% ; \nabla, 2 \% ; 0$, $3 \%, \triangle, 4 \%$. The concentration of Polypepton was $0.5 \%$.

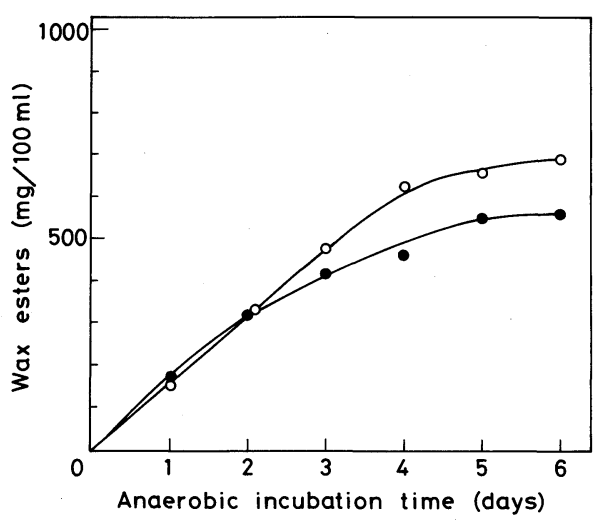

FIG. 4. Effect of the Glucose Concentration on the Accumulation of Wax Esters.

After aerobic cultivation for 3 days, anaerobic incubations were carried out with the supplementation of $1 \%$ oleic acid. The concentrations of glucose were: $\bigcirc, 3 \% ; 0,2 \%$. The concentration of Polypepton was $0.5 \%$. 
centration of $3 \%$, and the growth reached the stationary phase in about 4 days, with a yield of about $14.0 \mathrm{~g}$ dry cells/liter. Polypepton at a concentration of more than $1 \%$ delayed cell growth, a Polypepton concentration of $0.5 \%$

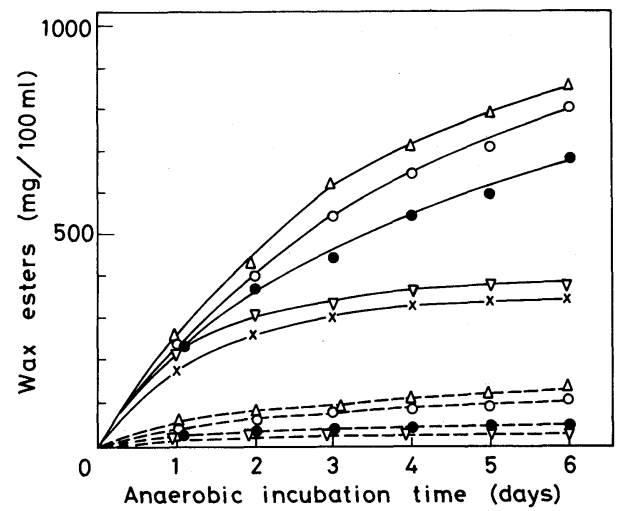

FIG. 5. Effect of the Oleic Acid Concentration on the Accumulation of Wax Esters.

The amounts of total wax esters (-) and oleyl oleate (---) were determined during anaerobic incubation. The concentrations of oleic acid were: $\times, 0 \%, \nabla, 0.3 \%$; $0.5 \% ; \bigcirc, 1.0 \% ; \triangle, 1.5 \%$. The concentrations of glucose and Polypepton were $3 \%$ and $0.5 \%$, respectively. being suitable for the cell growth.

Figure 4 shows the effect of the initial concentration of glucose on wax ester accumulation. Wax ester production in $3 \%$ glucose medium was higher than that in $2 \%$ glucose medium. As the cell growth in both media just before the shift to anaerobic conditions was almost the same (as shown in Fig. 3), it was supposed that the difference in the wax ester accumulation was due to the amount of acetylCoA resources such as paramylon in the cells and the residual glucose in the medium. From these results, the glucose-peptone medium, in which the concentrations of glucose and Polypepton were $3 \%$ and $0.5 \%$, respectively, was thought to be suitable for the wax ester production and was used throughout the following experiments.

The effect of the concentration of the supplemented oleic acid on wax wster accumulation is shown in Fig. 5. Oleyl oleate production was calculated by means of gas-liquid chromatography with the assumption that the $\mathrm{C}_{36}$ component consisted of only oleyl oleate. Wax

Table III. Wax Ester Production on Anaerobic Incubation with Supplementation of $1 \%$ of Various Unsaturated Fatty Acids

\begin{tabular}{|c|c|c|c|c|c|c|c|c|c|c|}
\hline Supplemented fatty acid & \multicolumn{2}{|c|}{ Oleic } & \multicolumn{2}{|c|}{ Linoleic } & \multicolumn{2}{|c|}{$\alpha$-Linolenic } & \multicolumn{2}{|c|}{ Ricinoleic } & \multicolumn{2}{|c|}{ None } \\
\hline Dry cell weight $(\mathrm{g} /$ liter $)$ & \multicolumn{2}{|c|}{15.7} & \multicolumn{2}{|c|}{14.6} & \multicolumn{2}{|c|}{14.2} & \multicolumn{2}{|c|}{14.6} & \multicolumn{2}{|c|}{12.0} \\
\hline Wax ester yield (g/liter) & & 7.7 & & 8.4 & & 6.9 & & 7.0 & & 2.8 \\
\hline \multirow{2}{*}{$\begin{array}{l}\text { Component of } \\
\text { wax esters }\end{array}$} & \multicolumn{10}{|c|}{ Relative amounts of wax esters $(\%)$} \\
\hline & Acyl & Alkoxy & Acyl & Alkoxy & Acyl & Alkoxy & Acyl & Alkoxy & Acyl & Alkoxy \\
\hline $11: 0$ & - & 0.2 & 0.2 & 0.3 & - & 0.5 & - & 0.6 & 0.2 & 0.5 \\
\hline $12: 0$ & 4.5 & 9.5 & 5.6 & 14.7 & 2.7 & 9.7 & 2.0 & 11.5 & 16.4 & 19.2 \\
\hline $13: 0$ & 1.6 & 3.3 & 2.2 & 5.4 & 3.0 & 10.9 & 4.6 & 10.6 & 16.5 & 16.8 \\
\hline $14: 0$ & 27.5 & 51.4 & 25.9 & 54.0 & 14.2 & 49.0 & 15.4 & 55.9 & 56.0 & 54.3 \\
\hline $15: 0$ & 0.4 & 2.8 & 0.3 & 0.9 & 0.8 & 7.7 & 0.4 & 7.3 & 2.0 & 1.8 \\
\hline $16: 0$ & 2.8 & 12.3 & 2.8 & 6.8 & 1.4 & 11.1 & 2.5 & 11.2 & 7.2 & 7.4 \\
\hline $17: 0$ & 0.7 & - & 0.7 & - & 0.3 & - & 0.5 & - & 1.4 & - \\
\hline $18: 0$ & 0.5 & 0.3 & - & - & - & - & - & - & 0.2 & - \\
\hline $16: 1$ & 0.8 & 0.2 & - & $\dot{-}$ & - & - & 0.2 & - & - & - \\
\hline $18: 1$ & 59.9 & 19.5 & 5.2 & 3.2 & 2.1 & 1.6 & 4.0 & 2.1 & - & - \\
\hline $18: 1 \mathrm{OH}^{*}$ & - & - & - & - & - & - & 60.7 & - & - & - \\
\hline $18: 2$ & 1.0 & - & 56.9 & 14.7 & 11.6 & 2.5 & 3.7 & 0.8 & - & - \\
\hline $18: 3$ & - & - & 0.2 & - & 63.9 & 7.0 & - & - & - & - \\
\hline $20: 1$ & 0.3 & 0.5 & - & - & - & - & - & - & - & - \\
\hline
\end{tabular}

* Ricinoleic or ricinoleyl. 
ester production during anaerobic incubation without oleic acid was almost completed in 3 days (shown in Fig. 1), while it continued even after 6 days' incubation in the presence of oleic acid at concentrations of more than $0.5 \%$. The rate of wax ester production increased with an increase in the amount of oleic acid added, and the amount of wax esters and oleyl oleate production also increased with it.

The results suggest that the uptake of oleic acid, which is immiscible with water, into the Euglena cells was not so fast, and that the uptake might be the rate-determining step in the unsaturated wax ester production.

\section{Production of liquid wax esters from various unsaturated fatty acids}

Wax esters were synthesized through anaerobic incubation for 6 days with supplementation of $1 \%$ of various unsaturated fatty acids (oleic, linoleic, $\alpha$-linolenic and ricinoleic acid). The results are shown in Table III.

The yields of wax esters separated from the lipid fractions by column chromatography were $7.7,8.4,6.9$ and $7.0 \mathrm{~g} /$ liter for the cells incubated with added oleic, linoleic, $\alpha$-linolenic and ricinoleic acid, respectively. The yields increased to about $50 \%$ of the dry cell weight for all of the incubations with fatty acids.

The unsaturated fatty acids, other than ricinoleic acid, were incorporated into the wax esters not only as the acyl moiety but also as the alkoxy one. This suggests that the fatty acyl-CoA reductase of Euglena may also reduce more or less these $\mathrm{C}_{18}$ unsaturated fatty acyl-CoAs except for the hydroxyl groupcontaining one, ricinoleoyl-CoA. Although the reducing enzyme seems to be less specific for these unsaturated fatty acyl-CoAs than for myristoyl-CoA, it is necessary to further elucidate the specificity of this enzyme for unsaturated fatty acyl-CoAs enzymatically.

The wax esters containing carbon-carbon double bonds, listed in Table III, are in the liquid state at room temperature. The physical properties of wax esters depend on their mo- lecular structure, that is, the carbon number, the number of unsaturations and the existence of substituent groups. The melting point of wax esters rises with an increase in carbon number and with a decrease in unsaturation, for example. From the results, it can be expected that various wax esters with a wide variety of physical properties can be prepared through the control of their molecular structures. The control of such factors as the carbon number, the number of unsaturations and the existence of substituent groups will be possible through the addition of unsaturated fatty acids favorable for the desired properties to the medium. The number of unsaturations could be controlled by the concentration of acetyl-CoA resources, such as paramylon in the cells and glucose in the medium.

Acknowledgments. The authors wish to thank Professor S. Kitaoka, Department of Agricultural Chemistry, University of Osaka Prefecture, for the gift of the Euglena strain and his valuable suggestions in this work. We also wish to thank Professor H. Yamada, Department of Agricultural Chemistry, Kyoto University, for his valuable advice on this work.

\section{REFERENCES}

1) J. E. Stewart and R. E. Kallio, J. Bacteriol., 78, 726 (1959).

2) R. L. Raymond and V. M. Jamison, Adv. Appl. Microbiol., 14, 93 (1971).

3) N. A. Krasilnikov and T. V. Koronelli, Mikrobiologia, 38, 757 (1969).

4) R. A. Makula, P. J. Lockwood and W. R. Finnerty, J. Bacteriol., 121, 250 (1975).

5) S. DeWitt, J. L. Ervin, D. Howes-Orchison, D. Dalietos and S. L. Neidleman, J. Am. Oil Chem. Soc., 59, 69 (1982).

6) H. Inui, K. Miyatake, Y. Nakano and S. Kitaoka, FEBS Lett., 150, 89 (1982).

7) H. Inui, K. Miyatake, Y. Nakano and S. Kitaoka, Agric. Biol. Chem., 47, 2669 (1983).

8) H. Inui and K. Miyatake, Kagaku to Seibutsu, 22, 422 (1984).

9) Th. J. De Boer and H. J. Backer, Org. Synthesis, Coll. Vol. 4, 250 (1963).

10) P. E. Kolattukudy, Biochemistry, 9, 1095 (1970).

11) L. E. Koren and S. H. Hutner, J. Protozool., 14, suppl. 17 (1967). 